\title{
Rules and Queries with Ontologies: a Unified Logical Framework
}

\author{
Enrico Franconi and Sergio Tessaris \\ Faculty of Computer Science, Free University of Bozen-Bolzano, Italy \\ lastname @inf . unibz.it
}

\begin{abstract}
In this paper we present a common framework for investigating the problem of combining ontology and rule languages. The focus of this paper is in the context of Semantic Web (SW), but the approach can be applied in any Description Logics (DL) based system. In the last part, we will show how rules are strictly related to queries.
\end{abstract}

\section{Introduction}

The need for integrating rules within the Semantic Web framework was clear since the early developments. However, up to the last few years, the research community focused its efforts on the design of the so called Ontology Layer. Nowadays, this layer is fairly mature in the form of Description Logics based languages such as OWL-Lite and OWL-DL, which are now among W3C recommendations.

One of the key features of SW ontology languages development is the attention to the computational properties of the main reasoning tasks. In particular, decidability is seen as one of the characteristics which should be preserved by these languages. This constraint led to the restriction of the expressivity of ontology language which can be heavy for certain applications (e.g. Web Services, or integration of information systems). The problem increasing the expressivity of SW ontology languages over the established Ontology Layer, together with the need of providing powerful query languages, directed the research towards the investigation of the possibility of combining OWL languages with Rules based languages.

In recent years, more research has been devoted towards the integration of different sorts of rule based languages on top of the ontology layer provided by the OWL languages and in more general terms on top of a generic DL, and this work already produced some proposals for extending OWL languages. However, these proposals comes from different research communities, and often are difficult to compare because of the diverse underlying semantic assumptions.

With this work we provide an unifying framework in which the existing (and future) proposals can be compared. Moreover, we present a thorough analysis

This work has been partially supported by the EU projects Sewasie, KnowledgeWeb, and Interop. 
of the main contributions, with a particular attention to their expressive power and restrictions to guarantee the decidability of key inference problems. By using our framework, we show that - under the appropriate restrictions - there are strong correspondences among the proposals. This enable us to isolate interesting fragments of the proposed languages in which we can compare the reasoning abilities.

We reckon that, since the early 90s, the Description Logics community produced several important results w.r.t. the problem of integrating DL languages and rules. For this reason we do not restrict our analysis to proposals in the context of Semantic Web. On the contrary, we show that a careful analysis of this body of work provides a valuable reference to explore the borders of expressivity and tractability of the combination of the two kinds of language.

In this paper we identify three different approaches: the axiom-based approach, the logic programming approach, and the autoepistemic approach. We provide an exact characterisation of the three approaches, together with a correspondence among relevant fragments in the three cases.

Moreover, we turn our attention at the problem of querying knowledge represented by means of an ontology web language. We show that there is a strong connection between rules and queries, and that our framework is able to capture this fundamental aspect of reasoning in the Semantic Web.

Our work on a common framework is directed to provide the Semantic Web community a tool which can be the basis for the discussion towards a common rule language for the Semantic Web. To this purpose we emphasise which, according to our opinion, is the correct semantics for a rule language. On this track we are currently working on a proposal for the OWL-Log language, which combines OWL ontology languages and rules. Our proposal takes into account the existing works, as well as establishes standards as RuleML.

\section{Rule-extended Knowledge Bases}

Let us consider a first-order function-free language with signature $\mathcal{A}$, and a description logic (DL) knowledge base $\Sigma$ with signature subset of $\mathcal{A}$.

In this paper we do not introduce any particular DL formalism. In our context, DL individuals correspond to constant symbols, DL atomic concepts and roles (and features) are unary and binary predicates in the case of a classical DL or a OWL language, and DL atomic $n$-ary relations correspond to predicates of arity $n$ in the case of a $\mathcal{D} \mathcal{L} \mathcal{R}$-like DL. Note that description logics with concrete data-types (such as OWL-Lite) are allowed as well.

A term is any constant in $\mathcal{A}$ or a variable symbol. If $R$ is a predicate symbol of arity $n$ and $t_{1}, \ldots, t_{n}$ are terms, $R\left(t_{1}, \ldots, t_{n}\right)$ is an atom, and an atom $R\left(t_{1}, \ldots, t_{n}\right)$ or a negated atom $\neg R\left(t_{1}, \ldots, t_{n}\right)$ are literals. A ground literal is a literal involving only constant terms. A set of ground literals is consistent if it does not contain an atom and its negation. If $l$ is a literal, $l$ or not $l$ are $N A F$-literals (negation as failure literals). DL atoms, DL literals, and DL NAF- 
literals are atoms, literals, and NAF-literals whose predicates belong to the DL signature. A rule $r$ may be of the forms:

$$
\begin{aligned}
h_{1} \wedge \ldots \wedge h_{\ell} & \leftarrow b_{1} \wedge \ldots \wedge b_{m} \\
h_{1} & :-b_{1} \wedge \ldots \wedge b_{m} \wedge \text { not } b_{m+1} \wedge \ldots \wedge \text { not } b_{n} \quad \text { (classical rule) } \\
h_{1} \wedge \ldots \wedge h_{\ell} & \Leftarrow b_{1} \wedge \ldots \wedge b_{m}
\end{aligned}
$$

where $h_{1}, \ldots, h_{\ell}, b_{1}, \ldots, b_{n}$ are literals. Given a rule $r$, we denote by $H(r)$ the set $\left\{h_{1}, \ldots, h_{\ell}\right\}$ of head literals, by $B(r)$ the set of body literals $\left\{b_{1}, \ldots, b_{n}\right\}$, by $B^{+}(r)$ the set of $N A F$-free body literals $\left\{b_{1}, \ldots, b_{m}\right\}$, and by $B^{-}(r)$ the set of $N A F$-negated body literals $\left\{b_{m+1}, \ldots, b_{n}\right\}$. We denote by $\operatorname{vars}\left(\left\{l_{1}, \ldots, l_{n}\right\}\right)$ the set of variables appearing in the literals $\left\{l_{1}, \ldots, l_{n}\right\}$. The distinguished variables of a rule $r$ are the variables that appears both in the head and in the body of the rule, i.e., $D(r)=\operatorname{vars}(H(r)) \cap \operatorname{vars}(B(r))$. A ground rule is a rule involving only ground literals. A rule is safe if all the variables in the head of the rule are distinguished. A DL rule is a rule with only DL literals. A set of literals is treeshaped if its co-reference graph is acyclic; a co-reference graph includes literals and variables as nodes, and labelled edges indicate the positional presence of a variable in a literal. An atomic rule is a rule having a single literal in the head. A set of rules is acyclic if they are atomic and no head literal transitively depends on itself; a head literal $h$ directly depends on a literal $l$ if there is an atomic rule $r$ with head $h$ and with $l$ part of the body $B(r)$. A set of rules is a view set of rules if each rule is atomic and no head literal belongs to the DL signature. A rule-extended knowledge base $\langle\Sigma, \mathcal{R}\rangle$ consists of a DL knowledge base $\Sigma$ and a finite set $\mathcal{R}$ of rules.

\section{The axiom-based approach}

Let us consider a rule-extended knowledge base $\langle\Sigma, \mathcal{R}\rangle$ restricted to only classical rules.

Let $I_{\Sigma}$ be a model of the description logics knowledge base $\Sigma$, i.e. $I_{\Sigma} \models \Sigma$. $I$ is a model of $\langle\Sigma, \mathcal{R}\rangle$, written $I \models\langle\Sigma, \mathcal{R}\rangle$, if and only if $I$ extends $I_{\Sigma}$ with the interpretation of the non-DL predicates, and for each rule $r \in \mathcal{R}$ then

$$
I \models \forall \mathbf{x}, \mathbf{y} \cdot \exists \mathbf{z} \cdot(\bigwedge B(r) \rightarrow \bigwedge H(r))
$$

where $\mathbf{x}$ are the distinguished variables of the rule $D(r), \mathbf{y}$ are the non distinguished variables of the body $(\operatorname{vars}(B(r)) \backslash D(r))$, and $\mathbf{z}$ are the non distinguished variables of the head $(\operatorname{vars}(H(r)) \backslash D(r))$.

Let us define now the notion of logical implication of a ground literal $l$ given a rule extended knowledge base: $\langle\Sigma, \mathcal{R}\rangle \models l$ if and only if $I \models l$ whenever $I \models\langle\Sigma, \mathcal{R}\rangle$. Note that the problems of DL concept subsumption and DL instance checking, and the problem of predicate inclusion (also called query containment) are all reducible to the problem of logical implication of a ground literal. Logical implication in this framework is undecidable, as it generalises the so-called recursive CARIN as presented in [Levy and Rousset, 1998]. Logical implication 
in an axiom-based rule extended knowledge base remains undecidable even in the case of atomic negation-free safe DL rules with a DL having just the universal role constructor $\forall R$. C. Note that logical implication in an axiom-based rule extended knowledge base even with an empty TBox in $\Sigma$ is undecidable (see, e.g., [Baget and Mugnier, 2002]).

In order to recover decidability, we reduce the expressivity of the approach in several ways; all the following restrictions disallow non DL predicates in the rules.

Theorem 1. 1. If we restrict the axiom-based approach to have only DL rules with tree shaped heads and bodies and without negated atomic roles, the problem of logical implication in the rule extended knowledge base is NEXPTIMEcomplete with $\mathcal{A L C} \mathcal{C I}, O W L$-Lite and $O W L-D L$ as the underlying description logics knowledge base language.

2. If in addition to the above conditions, constants are disallowed from the rules, the problem of logical implication in the rule extended knowledge base is

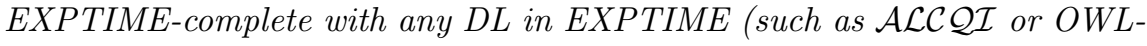
Lite) as the underlying description logics knowledge base language.

3. [Levy and Rousset, 1998]: If we restrict the axiom-based approach to have

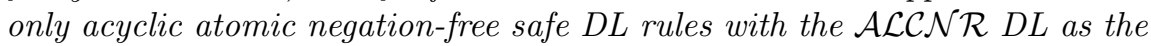
underlying description logics knowledge base language, the problem of logical implication is decidable in NEXPTIME.

The SWRL proposal [Horrocks and Patel-Schneider, 2004] can be considered as a special case of the axiom-based approach presented above. SWRL uses OWL-DL or OWL-Lite as the underlying description logics knowledge base language (which admits data types), but it restricts the rule language to safe rules and without negated atomic roles. From the point of view of the syntax, SWRL rules are an extension of the abstract syntax for OWL DL and OWL Lite; SWRL rules are given an XML syntax based on the OWL XML presentation syntax; and a mapping from SWRL rules to RDF graphs is given based on the OWL $\mathrm{RDF} / \mathrm{XML}$ exchange syntax. Logical implication in SWRL is still undecidable. The complexity results listed in Theorem 1 are applicable to SWRL as well.

Another way to make the axiom-based approach decidable is to reduce the expressivity of the DL, in order to disallow universal-like statements, while keeping rules cyclic.

In [Levy and Rousset, 1998] it is shown that logical implication is decidable with atomic negation-free safe DL rules with the simple DL containing conjunction, disjunction, qualified existential, least cardinality and primitive negation.

In [Calvanese et al., 2004] a proposal is made of a very simple knowledge representation language, which captures the fundamental features of frame-based formalisms and of ontology languages for the semantic web; the precise definition of the language can be found in [Calvanese et al., 2004]. In this setting, it can be shown that the negation-free axiom-based approach is decidable, and the problem of logical implication of a ground literal is in EXPTIME, and it is polynomial in data complexity. 
Conceptual graph rules [Baget and Mugnier, 2002] can be seen as a simple special case of an axiom-based rule extended knowledge base: CG-rules are negation-free, they do not have existential variables in the body, and $\Sigma$ is TBoxfree. Many decidable subclasses of CG-rules are special cases of the decidable cases presented above (but with $\Sigma$ having a TBox); in particular, decidability of range restricted $C G$-rules is the TBox-free special case stated above [Levy and Rousset, 1998] of atomic negation-free safe DL rules.

\section{The DL-Log approach}

Let us consider a rule-extended knowledge base $\langle\Sigma, \mathcal{R}\rangle$ where $\mathcal{R}$ is restricted to be a view set of lp-rules $\mathcal{P}$ (called program).

The non-DL Herbrand base of the program $\mathcal{P}$, denoted by $\mathcal{H} B_{\mathcal{P}^{-}}$, is the set of all ground literals obtained by considering all the non-DL predicates in $\mathcal{P}$ and all the constant symbols from $\mathcal{A}$. An interpretation $I$ wrt $\mathcal{P}$ is a consistent subset of $\mathcal{H} B_{\mathcal{P}^{-}}$. We say $I$ is a model of a ground literal $l$ wrt the knowledge base $\Sigma$, denoted $I \models_{\Sigma} l$, if and only if

$-l \in I$, when $l \in \mathcal{H} B_{\mathcal{P}}-$

$-\Sigma \models l$, when $l$ is a DL literal

We say that $I$ is a model of a ground rule $r$, written $I \models_{\Sigma} r$, if and only if $I \models_{\Sigma} H(r)$ whenever $I \models_{\Sigma} b$ for all $b \in B^{+}(r)$, and $I \not \nvdash_{\Sigma} b$ for all $b \in B^{-}(r)$. We denote with ground $(\mathcal{P})$ the set of rules corresponding to the grounding of $\mathcal{P}$ with the constant symbols from $\mathcal{A}$. We say that $I$ is a model of a rule-extended knowledge base $\langle\Sigma, \mathcal{P}\rangle$ if and only if $I \models_{\Sigma} r$ for all rules $r \in \operatorname{ground}(\mathcal{P})$; this is written as $I \models\langle\Sigma, \mathcal{P}\rangle$.

Let us define now the notion of logical implication of a ground literal $l$ given a rule extended knowledge base: $\langle\Sigma, \mathcal{P}\rangle \models l$ if and only if $I \models_{\Sigma} l$ whenever $I \models\langle\Sigma, \mathcal{P}\rangle$. In the case of a NAF-free program, as well in the case of a program with stratified NAF negation, it is possible to adapt the standard results of datalog, which say that in these cases the logical implication can be reduced to model checking in the (canonical) minimal model. So, if $I_{m}^{\mathcal{P}}$ is the minimal model of a NAF-free or stratified program $\mathcal{P}$, then $\langle\Sigma, \mathcal{P}\rangle \models l$ if and only if $I_{m}^{\mathcal{P}} \models_{\Sigma} l$.

In the case of an unrestricted program $\mathcal{P}$, an answer set semantics can be adopted to characterise logical implication. In this paper we do not define the semantics of unrestricted rule extended knowledge bases; for a precise account, please refer to [Rosati, 1999; Eiter et al., 2004].

Theorem 2. [Eiter et al., 2004]: The combined complexity of logical implication in a rule extended knowledge base with an EXPTIME-complete description logic

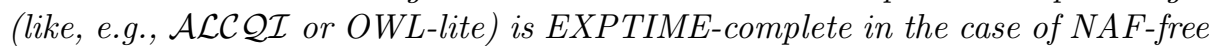
or stratified programs and it is NEXPTIME-complete in the unrestricted case. In a rule extended knowledge base with a NEXPTIME-complete description logic (like, e.g., $\mathcal{A L C Q \mathcal { O } O}$ or $O W L-D L)$ the complexity is NEXPTIME-complete in the case of $N A F$-free programs and it is $N P^{N E X P}$-complete in the case of stratified programs and in the unrestricted case as well. 
In addition, it is possible to prove that the problem of logical implication of a DL literal in a rule extended knowledge base is independent on the presence of the program $P$. This means that the DL knowledge base is unaffected by the rule system, which can be seen as built on top of the DL knowledge base.

The DL-Log approach was first introduced with AL-Log. The AL-Log approach [Donini et al., 1998b] is as a restriction of DL-Log. In fact, in AL-Log only view negation-free safe rules, whose DL predicates are only unary, with the $\mathcal{A L C} \mathrm{DL}$, are allowed. The complexity of logical implication is shown to be in NEXPTIME. [Rosati, 1999] extended AL-Log by allowing any DL predicate in the body of the rules. [Eiter et al., 2004] introduced DL-Log in the way we are presenting here.

An extension of DL-Log is the one where the recursive program is given a fixpoint semantics, which involves all individuals in the model, not only the ones in the Herbrand universe. In this extension, logical implication is undecidable with any DL having the ability to state at least atomic inclusion axioms between concepts [Calvanese and Rosati, 2003]. It can be shown that, in the fixpoint based semantics, the DL-Log approach can be reconstructed by adding, for each rule, a special non-DL unary $t o p_{\mathcal{H} B}$ atom for each variable appearing in each DL literal of the rule, thus constraining the DL variables to be in the Herbrand universe anyway. Note also that in the case of acyclic rules, the fixpoint semantics coincide with the axiom-based semantics.

It is worthwhile mentioning at the end of this section three additional recent works that relate DLs with lp-rules: DLP [Grosof et al., 2003] and [Motik et al., 2004; Swift, 2004]. In these papers it is shown how to encode the reasoning problem of a DL into a pure logic programming setting, i.e., into a rule extended knowledge base with a $\Sigma$ without TBox. In the case of DLP, this is accomplished by encoding a severely restricted DL into a NAF-free negation-free DL program. In the two latter approaches, the full power of disjunctive logic programming is needed to perform the encoding of quite expressive DLs, at the cost of an exponential blow-up in space of the encoding.

\section{The autoepistemic approach}

Let us consider a rule-extended knowledge base restricted to autoepistemic rules.

Let $I_{\Sigma}$ be a model, over the non empty domain $\Delta$, of the description logics knowledge base $\Sigma$, i.e. $I_{\Sigma} \models \Sigma$. Let's define a variable assignment $\alpha$ in the usual way as a function from variable symbols to elements of $\Delta$. A model of $\langle\Sigma, \mathcal{R}\rangle$ is a non empty set $M$ of interpretations $I$, each one extending a DL model $I_{\Sigma}$ with some interpretation of the non-DL predicates, such that for each rule $r$ and for each assignment $\alpha$ for the distinguished variables of $r$ the following holds:

$$
(\forall I \in M . I, \alpha \models \exists \mathbf{x} . \bigwedge B(r)) \rightarrow(\forall I \in M . I, \alpha \models \exists \mathbf{y} . \bigwedge H(r))
$$

where $\mathbf{x}$ are the non distinguished variables of the body $(\operatorname{vars}(B(r)) \backslash D(r))$, and $\mathbf{y}$ are the non distinguished variables of the head $(\operatorname{vars}(H(r)) \backslash D(r))$. 
Let us define now the notion of logical implication of a ground literal $l$ given a rule extended knowledge base: $\langle\Sigma, \mathcal{R}\rangle \models l$ if and only if

$$
\forall M .(M \models\langle\Sigma, \mathcal{R}\rangle) \rightarrow \forall I \in M .(I \models l)
$$

The autoepistemic approach was first introduced by [Donini et al., 1998a], with the goal of formalising the constraint rules implemented in many practical DL systems. Such rules, in fact, are simple to implement since they influence the ABox reasoning, but leave the TBox reasoning unaffected. These rules are also the basis of the recent formalisations of peer-to-peer systems [Franconi et al., 2003]. As shown in [Franconi et al., 2003], the autoepistemic semantics as defined above is equivalent to the context-based semantics of [Ghidini and Serafini, 1998], and to the use of the autoepistemic operator, as defined, e.g., in [Reiter, 1992]. Using the results in [Marx, 1999; Gabbay et al., 2003], we can show that logical implication is decidable in the case of a rule extended knowledge base with DL rules with tree shaped body and heads, with the $\mathcal{A L C}$ DL; the precise complexity bounds are still unknown.

\section{Queries}

We now introduce the notion of a query to a rule extended knowledge base, that includes a DL knowledge base, a set of rules, and some facts.

Definition 1. A query to a rule extended knowledge base is a (possibly ground) literal $q_{\mathbf{x}}$ with variables $\mathbf{x}$ (possibly empty). The answer set of $q_{\mathbf{x}}$ is the set of all substitutions of $\mathbf{x}$ with constants $\mathbf{c}$ from $\mathcal{A}$, such that the for each substitution the grounded query is logically implied by the rule extended knowledge base, i.e.,

$$
\left\{\mathbf{c} \text { in } \mathcal{A} \mid\langle\Sigma, P\rangle \models q_{[\mathbf{x} / \mathbf{c}]}\right\} \text {. }
$$

This definition of query is based on the notion of certain answer in the literature and it is very general. Given a $\Sigma$, we define query rule over $\Sigma$ as a set of view rules together with a query literal selected from some head. In this way we capture the notion of a complex query expressed by means of a set of rules on top of an ontology.

The definition of query given above encompasses the different proposals of querying a DL knowledge base appeared in the literature. An important special case of query rule is with view acyclic DL axiom-based rules, which is better known as conjunctive query if each head literal appears only in one head, or positive query otherwise. Quite importantly, this restriction includes the seminal body of work on query answering with conjunctive queries (or with positive queries) with the very expressive $\mathcal{D} \mathcal{L} \mathcal{R}$ description logic (which includes $\mathcal{A L C Q \mathcal { I }}$ ) summarised in [Calvanese et al., 2000]. In this context, logical implication is EXPTIME-hard and in 2EXPTIME; in the case of a fixed finite domain (closed domain assumption) logical implication becomes coNP-complete in data complexity [Calvanese et al., 2000]. Practical algorithms for query answering 
have been studied in [Tessaris et al., 2002]. A proposal targeted towards the semantic web languages has been presented in [Horrocks and Tessaris, 2002].

Recently, the Joint US/EU ad hoc Agent Markup Language Committee has proposed an OWL query language called OWL-QL [Fikes et al., 2003], as a candidate standard language, which is a direct successor of the DAML Query Language (DQL). The query language is not fully formally specified, however it can be easily understood as allowing for conjunctive queries with distinguished variables (called must-bind variables) and non distinguished variables (called don't-bind variables). In addition, may-bind variables apparently provide the notion of a possible answer as opposed to the certain answer which has been adopted in this paper. Query premises of OWL-QL allow to perform a simple form of local conditional query; this could be encoded as assertions in DL queries as introduced in [Eiter et al., 2004].

\section{Comparing the three approaches}

We first show in this section the conditions under which the three approaches coincide. This corresponds essentially to the case of negation-free view ruleextended knowledge bases with empty TBoxes. Note that this is the case of pure Datalog without a background knowledge base, for which it is well known that the three different semantics give rise to the same answer set.

Theorem 3. If we restrict a rule extended knowledge base with classical rules to view negation-free $D L$ rules with TBox-free $\Sigma$, a rule extended knowledge base with lp-rules to NAF-free negation-free DL programs with TBox-free $\Sigma$, and a rule extended knowledge base with autoepistemic rules to view negation-free $D L$ rules with TBox-free $\Sigma$, the semantics of the rule extended knowledge base with classical rules, with lp-rules, and with with autoepistemic rules coincide, i.e., the logical implication problem is equivalent in the three approaches.

The above theorem is quite strict and it fails as soon as we release some assumption. We will show this by means of few examples. Consider the following knowledge base $\Sigma$, common to all the examples:

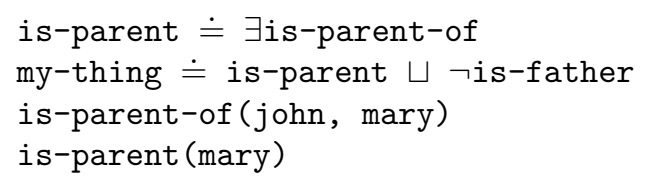

where we define, using standard DL notation, a TBox with the is-parent concept as anybody who is parent of at least some other person, and the concept my-thing as the union of is-parent and the negation of is-father (this should become equivalent to the top concept as soon as is-father becomes a subconcept of is-parent); and an ABox where we declare that John is a parent of Mary, and that Mary is parent of somebody.

Consider the following query rules, showing the effect of existentially quantified individuals coming from some TBox definition: 
$\mathrm{Q}_{\mathrm{ax}}(\mathrm{x}) \leftarrow$ is-parent-of $(\mathrm{x}, \mathrm{y})$

$\mathrm{Q}_{\mathrm{lp}}(\mathrm{x}):-$ is-parent-of $(\mathrm{x}, \mathrm{y})$

$\mathrm{Q}_{\mathrm{ae}}(\mathrm{x}) \Leftarrow$ is-parent-of $(\mathrm{x}, \mathrm{y})$

The query $Q_{a x}(x)$ returns $\{j \circ h n, \operatorname{mary}\}$; the query $Q_{l p}(x)$ returns $\{j \circ h n\}$; the query $Q_{a e}(x)$ returns $\{j o h n, \operatorname{mary}\}$.

Consider now the query rules, which shows the impact of negation in the rules:

$\mathrm{Q}_{\mathrm{ax}}(\mathrm{x}, \mathrm{y}) \leftarrow \neg$ is-parent-of $(\mathrm{x}, \mathrm{y})$

$\mathrm{Q}_{\mathrm{lp}}(\mathrm{x}, \mathrm{y}):-\neg$ is-parent-of $(\mathrm{x}, \mathrm{y})$

$\mathrm{Q}_{\mathrm{ae}}(\mathrm{x}, \mathrm{y}) \Leftarrow \neg$ is-parent-of $(\mathrm{x}, \mathrm{y})$

The query $Q_{a x}(\operatorname{mary}, j o h n)$ returns false; the query $Q_{l p}$ (mary, john) returns true; the query $Q_{a e}$ (mary, john) returns false.

Consider now the following alternative sets of rules, which show that autoepistemic rules, unlike the axiom-based ones, do not influence TBox reasoning:

is-parent $(\mathrm{x}) \leftarrow$ is-father $(\mathrm{x})$

$\mathrm{Q}_{\mathrm{ax}}(\mathrm{x}) \leftarrow \operatorname{my}-\operatorname{thing}(\mathrm{x})$

is-parent $(\mathrm{x}) \Leftarrow$ is-father $(\mathrm{x})$

$\mathrm{Q}_{\mathrm{ae}}(\mathrm{x}) \Leftarrow \operatorname{my}$-thing $(\mathrm{x})$

In the first axiom-based case, the query $Q_{a x}$ (paul) returns true; in the second autoepistemic case the query $Q_{a e}$ (paul) returns false (we assume that paul is an individual in $\Sigma$ ).

\section{Conclusions}

In this paper we have shown the differences and the similarities among three different semantics for rules in a knowledge base. We have also seen how queries can actually be seen as special case of rules.

We are currently working on the specification of the OWL-Log rule-extended knowledge base language in the DL-Log approach. OWL-Log is based on the various dialects of OWL (OWL-Lite and OWL-DL), and a syntax based on the interoperation between OWL and RuleML is planned.

\section{References}

[Baget and Mugnier, 2002] Jean-Francois Baget and Marie-Laure Mugnier. Extensions of simple conceptual graphs: the complexity of rules and constraints. Journal of Artificial Intelligence research (JAIR), 16:425-465, 2002.

[Calvanese and Rosati, 2003] Diego Calvanese and Riccardo Rosati. Answering recursive queries under keys and foreign keys is undecidable. In Proc. of the 10th Int. Workshop on Knowledge Representation meets Databases (KRDB 2003), pages 3-14. CEUR Electronic Workshop Proceedings, http://ceur-ws.org/Vol-79/, 2003. 
[Calvanese et al., 2000] Diego Calvanese, Giuseppe De Giacomo, and Maurizio Lenzerini. Answering queries using views over description logics knowledge bases. In Proc. of the 16th Nat. Conf. on Artificial Intelligence (AAAI 2000), pages 386-391, 2000.

[Calvanese et al., 2004] Diego Calvanese, Giuseppe De Giacomo, Domenico Lembo, Maurizio Lenzerini, and Riccardo Rosati. What to ask to a peer: Ontology-based query reformulation. In Proc. of the 9th Int. Conf. on the Principles of Knowledge Representation and Reasoning (KR 2004), 2004.

[Donini et al., 1998a] F. M. Donini, M. Lenzerini, D. Nardi, W. Nutt, and A. Schaerf. An epistemic operator for description logics. Artificial Intelligence, 100(1-2):225-274, April 1998.

[Donini et al., 1998b] Francesco M. Donini, Maurizio Lenzerini, Daniele Nardi, and Andrea Schaerf. $\mathcal{A} \mathcal{L}$-log: integrating datalog and description logics. Journal of Intelligent Information Systems, 10(3):227-252, 1998.

[Eiter et al., 2004] Thomas Eiter, Thomas Lukasiewicz, Roman Schindlauer, and Hans Tompits. Combining answer set programming with description logics for the semantic web. In Proc. of the International Conference of Knowledge Representation and Reasoning (KR'04), 2004.

[Fikes et al., 2003] Richard Fikes, Patrick Hayes, and Ian Horrocks. OWL-QL - A Language for Deductive Query Answering on the Semantic Web. Technical report, Knowledge Systems Laboratory, Stanford University, Stanford, CA, KSL-03-14, 2003.

[Franconi et al., 2003] Enrico Franconi, Gabriel Kuper, A. Lopatenko, and L. Serafini. A robust logical and computational characterisation of peer-to-peer database systems. In International VLDB Workshop On Databases, Information Systems and Peer-to-Peer Computing (DBISP2P'03), 2003.

[Gabbay et al., 2003] D.M. Gabbay, A. Kurucz, F. Wolter, and M. Zakharyaschev. many-Dimensional Modal Logics: Theory and Applications. Elsevier, 2003.

[Ghidini and Serafini, 1998] Chiara Ghidini and Luciano Serafini. Distributed first order logics. In Franz Baader and Klaus Ulrich Schulz, editors, Frontiers of Combining Systems 2, Berlin, 1998. Research Studies Press.

[Grosof et al., 2003] Benjamin N. Grosof, Ian Horrocks, Raphael Volz, and Stefan Decker. Description logic programs: Combining logic programs with description logic. In Proc. of the Twelfth International World Wide Web Conference (WWW 2003), pages 48-57. ACM, 2003.

[Horrocks and Patel-Schneider, 2004] Ian Horrocks and Peter F. Patel-Schneider. A proposal for an owl rules language. In Proc. of the Thirteenth International World Wide Web Conference (WWW 2004), 2004.

[Horrocks and Tessaris, 2002] Ian Horrocks and Sergio Tessaris. Querying the semantic web: A formal approach. In Proc. International Semantic Web Conference 2002 (ISWC-02), pages 177-191, 2002.

[Levy and Rousset, 1998] Alon Y. Levy and Marie-Christine Rousset. Combining Horn rules and description logics in CARIN. Artificial Intelligence, 104(1-2):165-209, 1998.

[Marx, 1999] Maarten Marx. Complexity of products of modal logics. J. Log. Comput., 9(2):197-214, 1999.

[Motik et al., 2004] Boris Motik, Ulrike Stattler, and Ullrich Hustadt. Reducing SHIQ description logic to disjunctive datalog programs. In Proc. of the International Conference of Knowledge Representation and Reasoning (KR'04), 2004.

[Reiter, 1992] Raymond Reiter. What should a database know? Journal of Logic Programming, 14(2,3), 1992.

[Rosati, 1999] Riccardo Rosati. Towards expressive KR systems integrating datalog and description logics: a preliminary report. In Proc. of the 1999 International Description Logics workshop (DL'99), pages 160-164, 1999. 
[Swift, 2004] Terrance Swift. Deduction in ontologies via ASP. In Proc. of LPNMR 2004, pages 275-288, 2004.

[Tessaris et al., 2002] Sergio Tessaris, Ian Horrocks, and Graham Gough. Evaluating a modular abox algorithm. In Proc. of the International Conference of Knowledge Representation and Reasoning (KR'02), pages 227-238, 2002. 